\title{
Dynamics of Nonspecific Adsorption of Insulin to Erythrocyte Membranes
}

\author{
Robert M. Fulbright ${ }^{1}$ and Daniel Axelrod ${ }^{1}$
}

Received October 7, 1992; revised May 3, 1993; accepted May 5, 1993

\begin{abstract}
Molecules may arrive at targets (receptors, enzymes, etc.) localized on a membrane surface by first adsorbing onto the surface and then surface diffusing to the targets. The flux rate of molecules arriving at targets via this mechanism depends on the surface diffusion coefficient of the molecules and, in some circumstances, on the adsorption/desorption kinetics. The technique of total internal reflection with fluorescence recovery after photobleaching (TIR-FRAP) was used here to study these rate parameters of fluorescein-labeled insulin (f-insulin) interacting with erythrocyte ghosts. Ghosts were adhered to polylysine coated slides for TIR illumination. Some ghosts became flattened and unsealed on the polylysine so that both extracellular and cytoplasmic sides of the membrane were openly exposed to the solution. An aluminum thin film between the polylysine and the fused silica of a slide quenched 'background' fluorescence from f-insulin adsorbed directly onto the polylysine. An interference fringe pattern from two intersecting and totally internally reflecting laser beams provided surface-selective excitation with a spatial variation of illumination intensity across a ghost for surface diffusion measurements. Measured characteristic values of desorption rate constants ranged from 0.043 to $270 \mathrm{~s}^{-1}$. According to a preexisting theoretical model, the largest desorption rate constant in this range would result in some increase in the total flux rate to a perfect sink target due to capture from the surface, provided that the surface diffusion coefficient was $\geqslant$ about $10^{-8} \mathrm{~cm}^{2} / \mathrm{s}$. However, based on TIR-FRAP measurements on our system, we estimate that the surface diffusion coefficient is less than about $5 \times 10^{-10} \mathrm{~cm}^{2} / \mathrm{s}$. The combination of novel techniques presented here may prove valuable to other workers seeking to make diffusive and chemical kinetic rate parameter measurements of biomolecules at biological cell membranes.
\end{abstract}

KEY WORDS: Total internal reflection fluorescence; surface diffusion; desorption kinetics; photobleaching recovery; hormone; reaction rates.

\section{INTRODUCTION}

Molecules from the bulk solution around a cell may, in principle, arrive at targets on the cell membrane surface via two routes. After diffusing through the bulk toward the surface, a molecule fortuitously may arrive directly at a target or it may adsorb, perhaps reversibly, at a nontarget region of the surface and then surface diffuse until arriving at a target. Under certain condi-

${ }^{1}$ Biophysics Research Division \& Department of Physics, University of Michigan, Ann Arbor, Michigan 48109. tions, this second route may increase the flux rate of molecules to the targets over that which would occur without surface diffusion [1-3]. In theory, the flux rate of bulk-dissolved molecules to perfect sink targets on the surface depends upon the rates of bulk diffusion, surface diffusion, and adsorption/desorption kinetics of the molecules [3]. In practice, very little is known about such "nonspecific" surface adsorption and diffusion in any in vivo system and whether it affects the binding rate to "specific" receptors.

Experimentally, adsorption/desorption rate con- 
stants and surface diffusion coefficients of fluorophorelabeled molecules interacting with a surface can be measured by total internal reflection fluorescence with fluorescence recovery after photobleaching (TIR-FRAP). In TIR, a laser beam is incident on a substrate-solution interface from the substrate side at a supercritical incidence angle. The evanescent field formed at the low refractive index (solution) side of the interface serves to selectively excite fluorophores proximal to the interface. In combination with FRAP as introduced by Thompson et al. [4], some of these fluorophores are irreversibly photobleached by a brief bright flash of the evanescent field, and the subsequent fluorescence recovery is monitored by an attenuated field as unbleached fluorophores from bulk solution and nonilluminated areas of the surface exchange with bleached ones by adsorption/desorption kinetics and surface diffusion. TIR-FRAP has been used previously to study the adsorption dynamics (kinetics and/or surface diffusion) of biological molecules at a variety of surfaces [5-9].

This paper describes a TIR-FRAP study of fluorescein-labeled insulin (f-insulin) molecules interacting with erythrocyte membrane. It is the first application of TIRFRAP to study biomolecular adsorption/desorption kinetics and surface diffusion at nonreceptor regions of a biological membrane. Human erythrocyte membrane was chosen for the study because it is readily available, easily prepared, and often regarded as a generic model of a cell membrane. There is a very low concentration of specific protein insulin receptors-about $14 / \mu \mathrm{m}^{2}$ on human erythrocyte membranes [10] - but we were not interested in studying the binding kinetics to these sites. Rather, we measure values of adsorption/desorption kinetic rate constants and surface diffusion coefficients of insulin interacting with the nonreceptor (also termed nonspecific) areas of the membrane. Insulin was chosen for the study because it is a fluorophore-labelable commercially available protein which might serve as a model of nonspecific adsorption of a biologically important agonist/hormone to nontarget regions of a cell membrane. In addition, fluorescein-labeled insulin associates with erythrocyte membrane at a sufficiently high surface concentration to give a usable fluorescence signal. In previous equilibrium adsorption experiments using TIRF, Sui $e t$ al. [11] found that fluorescein-labeled insulin does associate with artificial phospholipid membranes which are totally free of specific insulin receptors, particularly those membranes with a net negative charge.

Characteristic values of desorption rate constants measured here by TIR-FRAP ranged from 0.043 to 270 $\mathrm{s}^{-1}$. According to a preexisting theoretical model, the largest desorption rate constant in this range would result in some increase in the total flux rate to a perfect sink target due to capture from the surface, provided that the surface diffusion coefficient was $\geqslant 10^{-8} \mathrm{~cm}^{2} / \mathrm{s}$. However, based on the TIR-FRAP measurements on our system, we estimate that the surface diffusion coefficient is less than about $5 \times 10^{-10} \mathrm{~cm}^{2} / \mathrm{s}$.

Aside from the possible relevance of our results to understanding the kinetics of hormone diffusion to cell surface receptors, several of the techniques used here are novel modifications of preexisting approaches and may be of general interest. These techniques include a method for flattening erythrocyte ghosts onto a surface which exposes their external and cytoplasmic faces in distinct regions, the use of a thin aluminum substrate coating to suppress fluorescence from labeled insulin directly adsorbed to the substrate, and TIR-FRAP combined with a striped spatial pattern produced by optical interference.

\section{METHODS}

\section{Preparation of Glass Substrate}

Human erythrocytes have a negative surface charge which allows them to adhere to positively charged surfaces. In our case, the adherence must be very tight in order to withstand subsequent erythrocyte hemolysis. Therefore, the first task is to prepare highly positive glass surfaces by covalently attaching polylysine. The procedure we use is a modification of one used previously on microscopic glass spheres [12].

Poly-L-lysine (Sigma Chemical Co.; St. Louis, MO; MW 70,000-300,000) was covalently attached either to commercial grade or Suprasil 2 grade fused silica microscope slides (Heraeus Amersil, Inc.; Duluth, GA; 1 in. $\times 1$ in. $\times 1 \mathrm{~mm}$ ) or to regular glass microscope slides. Fused silica is generally better for faster timescale experiments where the millisecond duration luminescence of regular glass might interfere with postbleaching flash fluorescence observation.

Cleaning. Slides were cleaned in a loosely capped slide staining dish by exposing them to $10 \%$ nitric acid at approx. $90^{\circ} \mathrm{C}$ for 1 to $2 \mathrm{~h}$. Without removing them from the dish, the slides were rinsed eight times with $\mathrm{H}_{2} \mathrm{O}$ and three times with acetone. The dish was then loosely capped and placed in a $110^{\circ} \mathrm{C}$ oven for at least $12 \mathrm{~h}$.

Silanization. The slide staining dish was removed from the oven and allowed to cool to room temperature. A $3.5 \%$ (by volume) solution of 3aminopropyltriethoxysilane (Sigma) in anhydrous toluene was added to the dish until the slides were covered 
by at least a $1.25-\mathrm{cm}$ depth of solution. Toluene was made anhydrous by storage over indicating $\mathrm{CaSO}_{4}$ granules for at least $24 \mathrm{~h}$ and filtered through Whatman No. 5 filter paper just before use. Indicating $\mathrm{CaSO}_{4}$ granules were poured into the bottom of a large beaker and the beaker was partially filled with anhydrous toluene. The loosely capped dish containing the slides was put into the beaker and the beaker was capped with $\mathrm{Al}$ foil and placed into a preheated $85-90^{\circ} \mathrm{C}$ vacuum oven under an exhaust hood. The slide staining dish was put into the toluene-containing beaker so that the air surrounding the dish would be saturated with toluene. The slides were kept in the oven for 2-3 days. After the slides were removed from the oven they were individually rinsed three times in toluene and three times in acetone and then placed into a clean slide staining dish. These aminopropyl slides were stored under vacuum in a desiccator at least overnight before further use.

Succinylation. Each aminopropyl slide was dipped twice in $0.1 \mathrm{M}$ sodium acetate buffer, $\mathrm{pH} 5.0$, once in 95\% ethanol, twice in acetone, and once in anhydrous acetone, then placed into a clean slide staining dish. Acetone was made anhydrous by storage over $\mathrm{CaSO}_{4}$ granules (nonindicating) for at least $24 \mathrm{~h}$ and filtered through Whatman No. 5 filter paper just before use. A freshly made anhydrous acetone solution containing $\mathbf{5 0}$ g succinic anhydride per liter and 5\% pyridine (by volume) was added to the slide staining dish to a depth of at least $1.25 \mathrm{~cm}$ above the slides and the dish was loosely capped. $\mathrm{CaSO}_{4}$ granules were poured into the bottom of a large beaker and the beaker was partially filled with anhydrous acetone. The slide staining dish was placed in the beaker and the beaker was capped with $\mathrm{Al}$ foil. The beaker was placed in a preheated $50-55^{\circ} \mathrm{C}$ vacuum oven under an exhaust hood for 4 to $6 \mathrm{~h}$. After succinylation the slides were individually rinsed twice in $95 \%$ ethanol and twice in acetone. These succinylaminopropyl slides were stored in a clean slide staining dish under vacuum in a desiccator at least overnight before further use.

Polylysination. For each succinylaminopropyl slide that was to be polylysinated, $15 \mathrm{mg}$ poly-L-lysine was dissolved in $61 \mu \mathrm{l} \mathrm{H}_{2} \mathrm{O}$ and $31 \mu \mathrm{l} 4 \mathrm{M}$ pyridine. Each slide was dipped in $\mathrm{H}_{2} \mathrm{O}$ and placed in its own petri dish and $90 \mu \mathrm{l}$ of the poly-L-lysine solution was dropped onto each slide. Then $61 \mu \mathrm{l} 5.2 \mathrm{M}$ pyridine- $\mathrm{HCl}, \mathrm{pH} 5.0$, was dropped onto each slide. Finally, a total of $15.3 \mu \mathrm{l}$ freshly made 1.0 M 1-ethyl-3(3-dimethylaminopropyl)-carbodiimide- $\mathrm{HCl}$ (Sigma) was dropped on each slide in three aliquots of $5.1 \mu \mathrm{l}$. The petri dishes were covered and taped to a mixer, where they were gently shaken for approx. $2 \mathrm{~h}$. Each slide was then individually rinsed once in $\mathrm{H}_{2} \mathrm{O}$, twice in $4 \mathrm{MNH}_{4} \mathrm{Cl}$, and five times in $\mathrm{H}_{2} \mathrm{O}$, then placed polylysine side up in its own clean petri dish and allowed to air-dry. The petri dishes containing the slides were capped, labeled, and stored under vacuum in a freezer until the slides were needed for experiments.

\section{Attachment and Hemolysis of Erythrocyte Membranes at Substrate}

One drop of fresh human blood was mixed into 2 $\mathrm{ml}$ of Hanks' balanced salt solution without calcium, magnesium, and phenol red (HBSS; GIBCO Laboratories Life Technologies, Inc.; Grand Island, NY). The suspension was then centrifuged, washed with more HBSS, and recentrifuged. The pellet was then resuspended in about $4 \mathrm{ml}$ of HBSS, and $0.5 \mathrm{ml}$ of the suspension was further diluted into $3.5 \mathrm{ml}$ of HBSS.

A polylysine-coated slide was prewet with HBSS and the excess drained. Then a few drops of the diluted erythrocyte solution was applied to the slide for 15-30 min (but not longer), and the slide was placed in HBSS. To hemolyze the adhered erythrocytes, the slide was taken from the HBSS rinse, put into pure $\mathrm{H}_{2} \mathrm{O}$ for 6 to $6.5 \mathrm{~min}$, and then once again placed in an HBSS rinse.

\section{Characterization of the Erythrocyte Membrane Configuration}

We infer from various fluorescence labeling experiments that many (sometimes most) of the substrate-adhered membranes produced by the above procedure assume a flattened configuration (depicted in Fig. 1) with a characteristic rip that exposes the cytoplasmic and external faces in distinct regions. These separate labeling experiments used a lectin [rhodamine-labeled wheat germ agglutinin (r-WGA; Vector Labs; Burlingame, CA) ], a carbocyanine lipid probe dye $\left[1,1^{\prime}\right.$-dioctadecyl-3,3,3', $3^{\prime}$ tetramethylindocarbocyanine (diI; Molecular Probes; Eugene, OR)], and an antibody to a cytoskeletal protein (anti-spectrin, a gift from Drs. George Brewer and Jon Aster).

Figure 2 shows epiillumination photomicrographs of fluorescence from r-WGA (which binds only to the external surface at certain sugar residues). In Fig. 2a, the whole erythrocytes were first exposed to r-WGA $(\sim 5$ $\mu \mathrm{g} / \mathrm{ml})$, rinsed in HBSS, adhered to the substrate, and then hemolyzed as above. This is "prelabeling." In Fig. $2 b$, the cells were first adhered to glass, then labeled with r-WGA, rinsed, and hemolyzed. This is "postlabeling." Taken together, Fig. 2 clearly shows that a "crescent"-shaped region consists of a membrane whose extracellular side is exposed to the solution. The prela- 


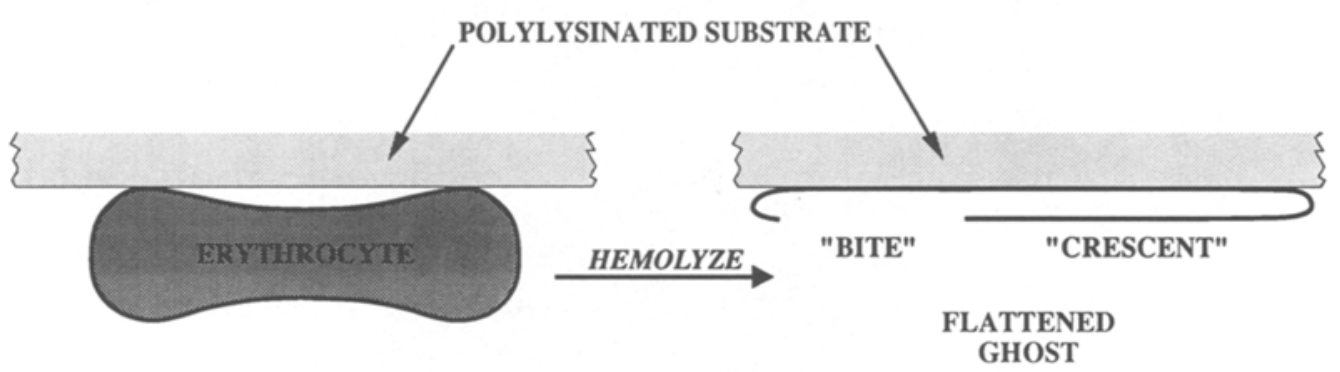

Fig. 1. Erythrocyte adhered to a polylysine-coated substrate is hemolyzed by osmotic shock, which collapses it to the substrate. We hypothesize that the membrane layer farthest from the substrate has a rip or hole in it so that the membrane near the substrate exposes its cytoplasmic surface to the solution (the "bite" region). Elsewhere (the "crescent" region), the extracellular side of the more distal membrane is exposed to the solution.
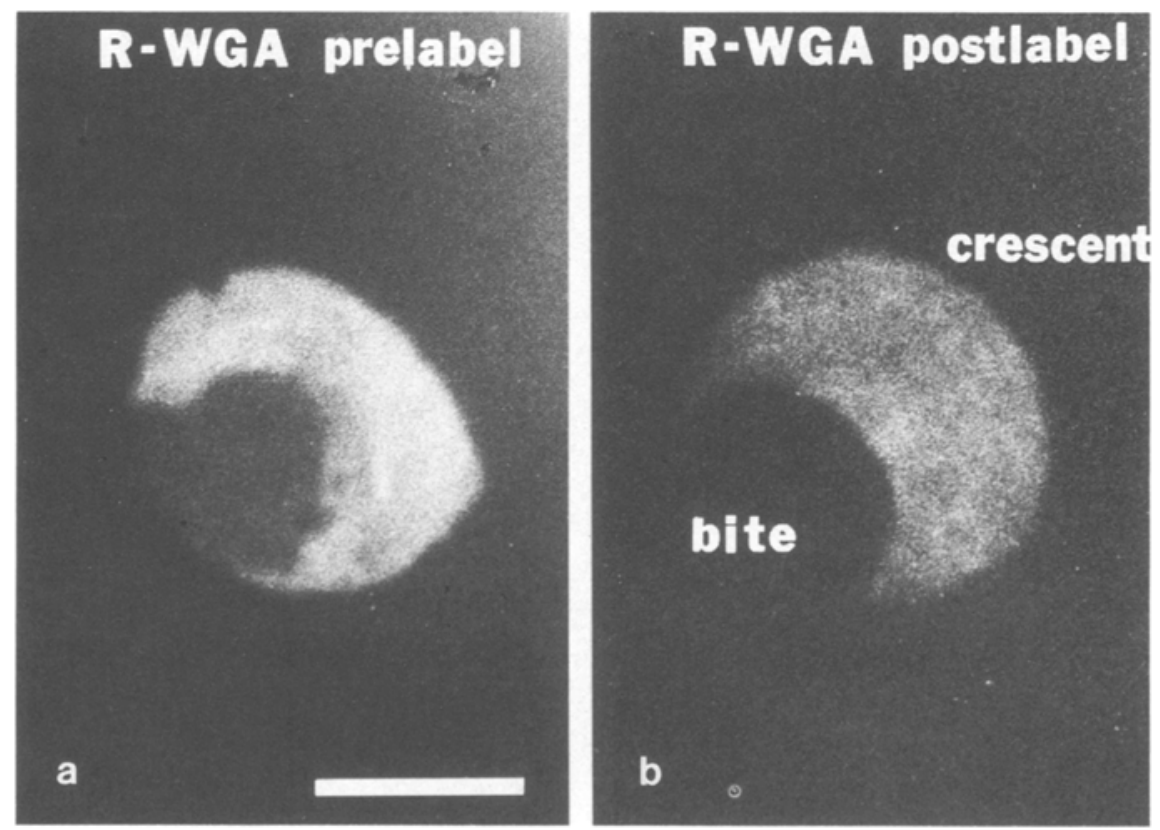

Fig. 2. (a) r-WGA-prelabeled flattened erythrocyte ghost. Erythrocytes were exposed to r-WGA, adhered to the polylysine slide, and then hemolyzed. (b) r-WGA-postlabeled flattened erythrocyte ghost. Erythrocytes were adhered to the polylysine slide, exposed to r-WGA, and then hemolyzed. In both cases, a "crescent" region is visible, but only in the prelabeled case is the "bite" region also visible. A 100×, 1.20-NA, Leitz waterimmersion objective was used in the epiillumination mode-5 $\mu \mathrm{m}$.

beled sample (Fig. 2a) confirms that the "bite"-shaped region partly surrounded by the crescent also contains a membrane. The dimmer fluorescence in the bite is consistent with the inference that it contains just one membrane layer instead of two, as conjectured in Fig. 1. Since the bite region is dark in the postlabeled sample, Fig. 2b, evidently the membrane in the bite region exposes only a cytoplasmic surface to the solution and apposes the substrate too closely for WGA to diffuse between the membrane and the substrate.
As would be expected from this configuration, samples that are adhered, hemolyzed, and then labeled very last show completely dark bites (not shown), much as in Fig. 2b. Dil-labeled samples (not shown) show bright crescents with substantial but dimmer bite labeling, qualitatively like Fig. 2a, again confirming that the bite contains a membrane.

Figure 3 shows a sample adhered to the substrate and hemolyzed in the usual manner but subsequently exposed to sheep antispectrin (a gift from Drs. Jon Aster 


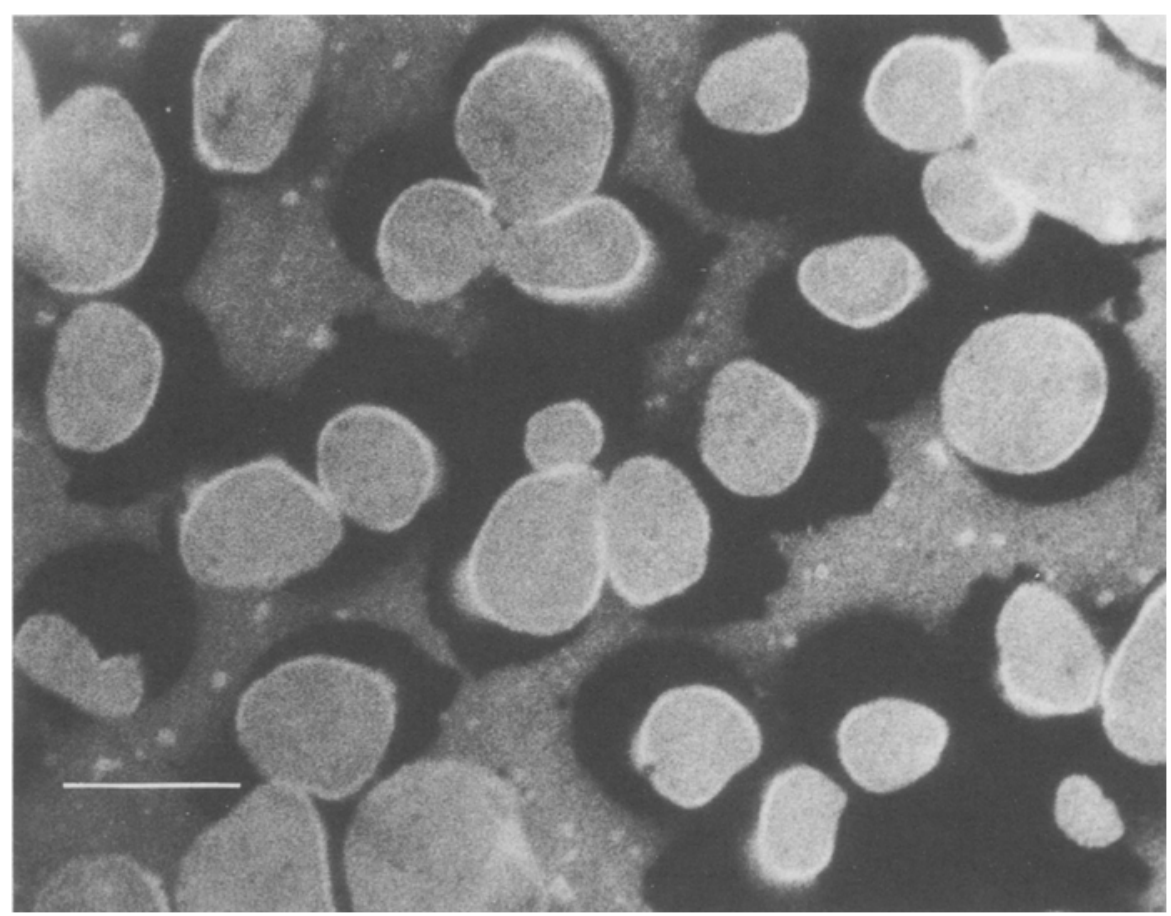

Fig. 3. Flattened erythrocyte ghosts labeled with antispectrin antibody. Erythrocytes were adhered to the polylysine slide, hemolyzed, exposed to sheep antispectrin, rinsed, and then exposed to a fluorescein anti-sheep second antibody. In contrast to the $r$-WGA labeling in Fig. 2 , here the "bite" region is much brighter than the "crescent." This photo was taken under TIR illumination with a 50×, 1.00-NA, water-immersion objective. Space bar, $5 \mu \mathrm{m}$.

and George Brewer) at $\sim 200 \mu \mathrm{g} / \mathrm{ml}$ for $\sim 30 \mathrm{~min}$, rinsed, exposed to fluorescein-labeled anti-sheep $(\sim 200 \mu \mathrm{g} / \mathrm{ml}$ for $\sim 20 \mathrm{~min}$ ), and then rinsed again. Note that the bite regions of the ghosts show very bright fluorescence. This again confirms the presence of a membrane in the bite region and suggests that its cytoplasmic side is exposed to the solution. The polylysinated surface of the slide is also labeled to some extent but not nearly as heavily as the bite regions. The "crescent" regions are very dark, suggesting that the conjectured two membrane layers in the crescent regions are apposed too closely for access by the antibodies.

\section{Quenching "Background" Fluorescence with an Aluminum Thin Film}

In the TIR-FRAP experiments discussed here, we examine reversible and rather weak binding of fluorophore-labeled insulin to the flattened erythrocyte ghost membrane in the crescent region, in contrast to the irreversible and strong binding of the WGA, diI, and antispectrin used to characterize the morphology. In fact, the adsorption of fluorescein-insulin to the membrane "crescent" is substantially weaker than its adsorption to the neighboring polylysine coated substrate. Because insulin is a rather small protein, it is possible that fluorescein-insulin could penetrate underneath the erythrocyte, bind to the polylysine, and overwhelm the fluorescence originating from membrane-adsorbed insulin. Therefore, we employed a novel optical procedure intended to selectively quench the fluorescence of insulin directly adsorbed to the polylysine. The procedure is based upon the high rate of nonradiative energy transfer from an excited fluorophore to a nearby metal surface. The rate of transfer decreases rapidly with increasing fluorophore distance from the metal and is usually significant only in the first $100 \AA[13-17]$. This highly local quenching effect can thereby suppress the fluorescence of $f$-insulin adsorbed directly onto the surface while retaining the fluorescence of f-insulin spaced away from the surface by two membrane thicknesses in the "crescent" region.

A thin ( 300 to $350 \AA$ ) film of $A l$ was vacuum evaporated onto fused silica microscope slides and poly-Llysine was coupled to the $\mathrm{Al}$, as follows. Slides (precleaned with nitric acid) were further cleaned in a plasma cleaner (Harrick Scientific Corp.; Ossining, NY) at a 
high $\mathrm{RF}$ level for at least $15 \mathrm{~min}$. The slides were then placed in a high-vacuum evaporator (Denton Vacuum, Inc.; Cherry Hill, NJ) and a measured quantity of $\mathrm{Al}$ wire was completely evaporated to form a reproducible coating thickness on the slides.

After evaporation, slides were kept under vacuum in the evaporator until it was time to begin silanization, the first chemical step to covalent polylysine linkage. A $0.5 \%$ (by volume) solution of 3-aminopropyltriethoxysilane in ethanol with 1.6 water molecules per silane molecule was made in a slide staining dish. The slides were quickly and completely submerged into the slide staining dish solution. After 4-8 min the solution was poured out and the dish containing the slides was rinsed out at least eight times with $100 \%$ ethanol. The dish was then loosely capped and stored under vacuum in a desiccator at least overnight before further use.

The next step, succinylation of the Al-coated slides, was identical to that used for slides without Al. The final step, polylysination, was also identical to that used for slides without $\mathrm{Al}$ except that (1) the poly-L-lysine solution was mixed with the $5.2 \mathrm{M}$ pyridine- $\mathrm{HCl}$, pH 5.0, before these solutions were dropped on the slides and (2) the reaction was allowed to proceed for only 15-20 min after all the solutions had been put on the slides. If the reaction was allowed to proceed for much longer than this, the $\mathrm{Al}$ coatings were so badly damaged that the slides were unusable. As with slides without $\mathrm{Al}$, the petri dishes containing the slides were capped, labeled, and stored under vacuum in a freezer until the slides were needed for experiments.

Used Al slide substrates could be reused after a thorough cleaning. Slides were wiped and rinsed in ethanol, exposed to $20 \mathrm{mM} \mathrm{NaOH}$ until the Al films dissolved (usually less than $5 \mathrm{~min}$ ), rinsed with $\mathrm{H}_{2} \mathrm{O}$, and then treated to the nitric acid cleaning step as above.

The adherence of flattened erythrocytes to Al slides prepared in this manner and the optical quenching of Alproximal fluorophores are shown in the TIRF photomicrographs of Fig. 4, both without (a) and with (b) an $\mathrm{Al}$ film. Note that the Al film effectively quenches fluorescence of "background" fluorophores directly adsorbed onto the polylysinated $\mathrm{Al}$ while preserving fluorescence from fluorophores adsorbed to the ghost crescents.

\section{Photodamage and Sample Deoxygenation}

In many TIRF experiments involving a fluorophorelabeled protein in equilibrium between a bulk dissolved and a surface adsorbed state, we have found that exposure to dim TIR excitation induces a continual slow increase in surface fluorescence. (At brighter illumination,
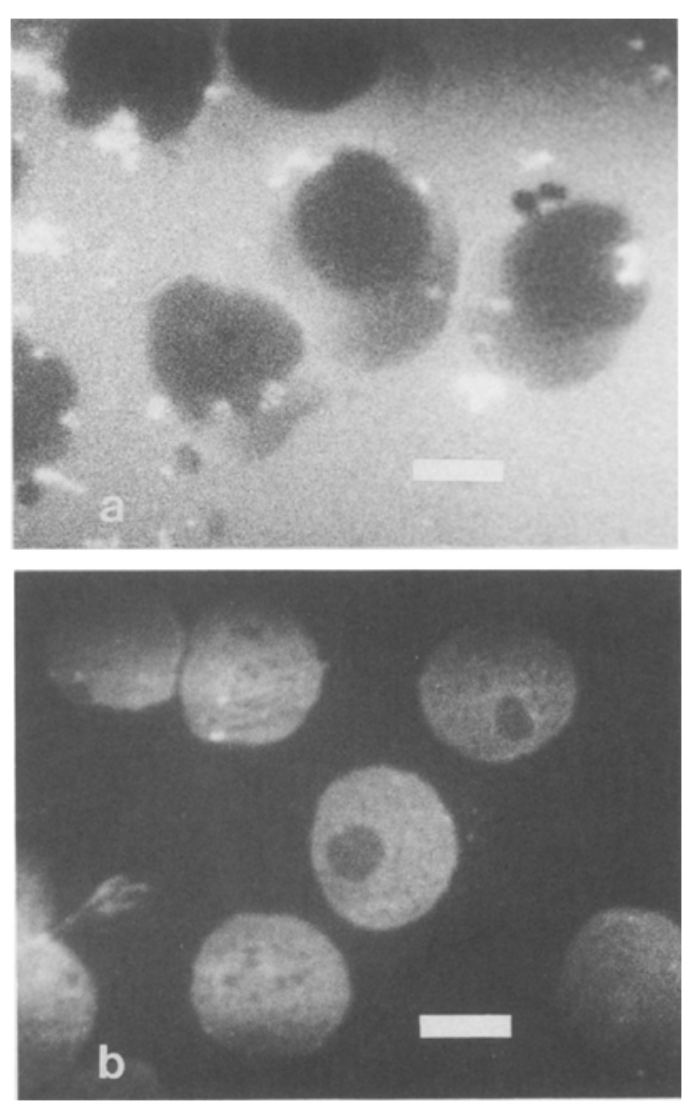

Fig. 4. Quenching of near-substrate fluorescence by an aluminum film coated onto the substrate. These are TIRF photos (a) on bare and (b) Al-coated polylysine-treated fused silica surfaces. In each case, the ghosts are in continuous contact with the same concentration of fluorescein epidermal growth factor solution containing some free fluorescein. These photos were taken with a $100 \times, 1.20-\mathrm{NA}$, waterimmersion objective. Space bar, $5 \mu \mathrm{m}$.

the surface fluorescence can be made to decrease by photobleaching as expected.) The rate of the slow increase seems to depend on the fluorophore type, with tetramethylrhodamine-labeled insulin showing the effect more severely than fluorescein-labeled insulin on the flattened erythrocyte samples. In general, this effect is not seen in other kinds of TIRF-illuminated samples, in which the adsorption to the surface is irreversible and the bulk concentration is virtually zero.

We conjecture that the slow fluorescence increase is due to cross-linking of excited fluorophores on the surface with free molecules containing fluorophores in the nearby bulk, thereby leading to a pileup of labeled protein on the surface. Studying a possibly analogous process, Sheetz and Koppel [18] found that visible light irradiation of fluorescein-labeled concanavalin $\mathrm{A}$ bound 
to the outside of resealed erythrocyte ghosts caused crosslinking of as much as $50 \%$ of the membrane proteins. Irradiation of unlabeled membranes or membranes labeled with unlabeled concanavalin A exhibited less than $10 \%$ membrane protein aggregation. Sheetz and Koppel also found that reducing the concentration of $\mathrm{O}_{2}$ in the sample prior to laser irradiation decreased the extent of protein cross-linking. Their results led them to speculate that reaction between the excited triplet state of the dye and $\mathrm{O}_{2}$ created an excited singlet form of oxygen $\left({ }^{1} \mathrm{O}_{2}\right)$ [19]. This ${ }^{1} \mathrm{O}_{2}$ then initiated reactions resulting in the cross-linking. Following their lead, we reduced the $\mathrm{O}_{2}$ content in our samples (by exposing them to $\mathrm{N}_{2}$ ) and found that this eliminated the gradual increase in fluorescence.

\section{Airtight Sample Chamber with Nitrogen Gas Flow- Through}

The requirement for continual suppression of oxygen dictated the preparative protocols and the sample chamber design. The airtight sample chamber with ports for $\mathrm{N}_{2}$ flow-through, designed for use with an inverted microscope and TIR sample illumination, is depicted in Fig. 5. The sample substrate glass forms the roof of the chamber, with the flattened ghosts facing downward into the solution. An almost-hemispherical fused silica prism (Harrick Scientific Corp.; Ossining, NY), which guides the laser beam into the slide for TIR illumination, is optically coupled to the sample slide by a thin layer of glycerin. The glass coverslip floor of the chamber is spaced away from the roof by about $0.1 \mathrm{~mm}$. Fluorescence from the ghosts propagates downward through the solution and coverslip floor and is collected by the microscope objective below the chamber. For standard epiillumination, the laser beam can be sent up through the objective.

The sample chamber, with its flattened hemolyzed erythrocytes on the $\mathrm{Al}$-coated substrate, was assembled in a glove bag under $\mathrm{N}_{2}$ gas, with silicone high-vacuum grease sealing the junctions. The volume of deoxygenated fluorescein-insulin solution $(200 \mu \mathrm{l}$; see preparation procedure below) used in the chamber formed a meniscus near the center of the slide, and moisturized pure $\mathrm{N}_{2}$ at $\sim 22^{\circ} \mathrm{C}$ flowed freely around it rather than bubbling through it. For details of the assembly procedure, see Fulbright [20].

\section{Fluorescein-Insulin Solution Used in TIR-FRAP Experiments}

In TIR-FRAP experiments which were designed to study the interaction of a hormone with a cell membrane, the labeling solution used was approximately $50 \mu M$ (monomer concentration; $0.31 \mathrm{mg} / \mathrm{ml}$ ) fluorescein-labeled bovine insulin (f-insulin; Sigma Chemical Co.; St. Louis, MO; Cat. No. I 2383) in Hanks' balanced salt solution without calcium, magnesium, and phenol red (HBSS; GIBCO Laboratories Life Technologies, Inc.; Grand Island, NY; $\mathrm{pH} 7.4$ ). This concentration was used

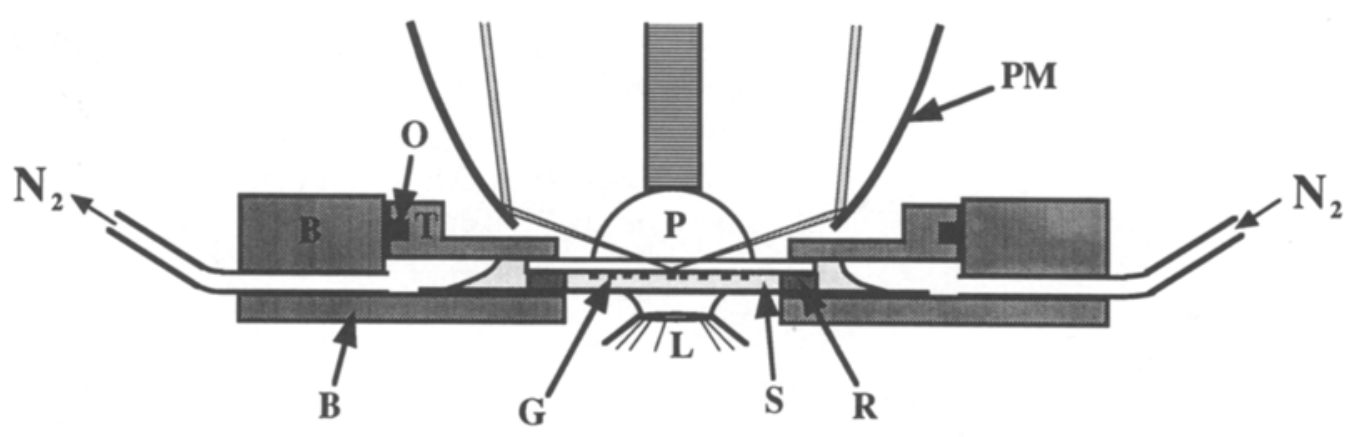

Fig. 5. Sample chamber, depicted in longitudinal cross section through its center. For clarity, the vertical dimensions of some of the components shown are exaggerated relative to the other components. The chamber's two main components are a top piece (T) and a bottom piece (B), which were machined from Lucite acrylic plastic. The hole in the bottom is covered with a thickness No. 1 cover glass glued to the bottom piece with RTV silicone rubber adhesive sealant. The $\mathrm{N}_{2}$ entry and exit ports are stainless-steel hypodermic needles glued into place with RTV silicone rubber adhesive sealant. A glass slide, with the erythrocyte ghosts $(G)$ adhered to its downward-facing surface, was sealed to the top piece of the chamber by silicone high-vacuum grease. Two Teflon spacer half-rings (R) $(0.1 \mathrm{~mm}$ thick) separated the glass slide from the cover glass of the chamber bottom. Two half-rings were used instead of one whole ring so that the solution (S) was more directly exposed to the $\mathrm{N}_{2}$ as it flowed through the chamber. The seal between the top and the bottom pieces of the chamber was made with a silicone high-vacuum greased $\mathrm{O}$-ring $(\mathrm{O})$. The top piece of the chamber was held securely to the bottom piece with screw-tightened clamps and was equipped with small handles (not shown) to remove the top piece easily from the bottom piece. Other abbreviations are paraboloid mirror (PM), prism (P), and objective lens (L). 
because it was high enough to give a usable fluorescence signal from nonspecific binding to "crescent" regions. In the dark at room temperature, $\mathrm{f}$-insulin was dissolved in enough HBSS to yield a $200 \mu M$ solution. This solution was then filtered through a $0.22 \mu \mathrm{m}$ pore size filter (Millex-GV $\mathrm{G}_{4}$; Millipore Corp.; Bedford, MA). This stock solution was stored at $4-5^{\circ} \mathrm{C}$ in the dark. When needed for experiments, $50 \mu \mathrm{l}$ of the stock solution was added to $150 \mu$ l of HBSS to make the $50 \mu \mathrm{M}$-insulin.

The method referenced by Sigma for labeling insulin with fluorescein was that of Tietze et al. [21]. According to Sigma, their product contains approx. 1.2 moles of fluorescein per mole of insulin monomer and less than $5 \%$ of free fluorescein as compared to bound fluorescein. In our final concentration of $50 \mu M$, singly labeled $f$-insulin and doubly labeled f-insulin would constitute roughly about 40 and $10 \mu M$, respectively. The fluorescein binds primarily to the terminal amino group of the phenylalanine residue of the $\mathrm{B}$ chain and secondarily to the terminal amino group of the glycine residue of the A chain $[21,22]$. Binding also occurs in triply labeled $\mathrm{f}$-insulin at the $\epsilon$-amino group of lysine (B chain) [22]. Tietze et al. [21] and Bromer et al. [22] found that their preparations of f-insulin retained $60-80$ and $40 \%$, respectively, of the biological activity of the native hormone.

In $0.11 \mathrm{M}$ phosphate buffer at $\mathrm{pH} 7.4-7.6$, singly labeled $\mathrm{f}$-insulin is a hexamer at a concentration of 100 $\mu M$ and doubly labeled $\mathrm{f}$-insulin is a dimer [23]. At $\mathrm{pH}$ 7.4, at concentrations $\leq 5 \mu M$, f-insulin (mono-, di-, and trisubstituted) is a monomer [24]. Circular dichroism and absorbance spectra taken as a function of concentration at $\mathrm{pH} 7.4$ and correlated with sedimentation data seem to indicate that our $\sim 40 \mu M$ population of mono-f-insulin would be a mixture of monomers, dimers, and hexamers, and our $\sim 10 \mu M$ di-f-insulin would be predominantly a population of monomers [24].

\section{Sample Illumination}

The laser beam used for both the probe and the bleach was passed through an acoustooptic modulator (AOM; IntraAction Corp.; Bellwood, IL), the first-order diffraction of which could be varied rapidly in intensity by a computer-generated bleaching pulse. The bleach intensity to probe intensity ratio for most TIR-FRAP experiments was in the range of 6000 to 8000 . The total power incident on the aluminum thin film during the bleaching flash is estimated to have been $0.014 \mathrm{~W}$, spread over an approximately elliptical area of $\sim 1000 \mu \mathrm{m}^{2}$.

Two types of TIRF illumination were used-single beam and double beam - the latter producing a fringe pattern by interference. A single-beam pattern produced a large area of approximately uniform illumination over an adhered ghost. The only significant postbleach recovery mechanism in such a case is adsorption/desorption kinetics. The double-beam fringe pattern superimposes on this area a finely spaced striped pattern. In this case, an additional recovery mechanism-surface diffusion across the stripes-may speed the recovery relative to the single beam case.

Figure 6 is a schematic diagram of the sample illumination optics used for TIR-FRAP experiments; it consists basically of two coherent beams split from the same laser, reflecting at a parabolic mirror, and recombining at a supercritical incidence angle at the sample plane, located near the focus of the parabolic mirror. By simply blocking one of the beams, we produce singlebeam illumination with no fringe pattern.

In principle, the fringe pattern period may be adjusted by varying the angle of intersection of the two laser beams at the sample. The angle of intersection is determined primarily by the separation of the two beams at the paraboloid mirror, which in turn is determined by the positions and choice of lenses L3 and L4 in Fig. 6.

Figure 7 is a photomicrograph of the fluorescence from f-insulin ( $\sim 50 \mu M$ in bulk solution) adsorbed onto a ghost under (TIR) fringe pattern illumination. The fringe pattern period is $(0.904 \pm 0.021) \mu \mathrm{m}$. We assumed the same fringe pattern period in all relevant TIR-FRAP experiments; its constancy was confirmed from a photographic image of each ghost used for data aquisition.

\section{Fluorescence Collection}

For TIR-FRAP, the cells were viewed through the $50 \times$, 1.0-NA water immersion objective of a Leitz Diavert microscope. An image plane diaphragm in the photometer unit between the microscope and the photomultiplier (Hamamatsu R943-02) ensures that fluorescence from only a specific region of the sample is detected. The aperture of the image plane diaphragm is adjusted by eye to be effectively a square of side length equal to two spatial periods of the illuminating fringe pattern. This size is small enough so that fluorescence could be collected exclusively from the "bite" or "crescent" region of a ghost. Ideally, with the aperture width spanning an integral number of fringe spatial periods and oriented squarely with the fringe pattern, the expected theoretical form of fluorescence recovery after photobleaching is simplified and independent of the relative position of the aperture and fringe pattern. Figure 7 shows 


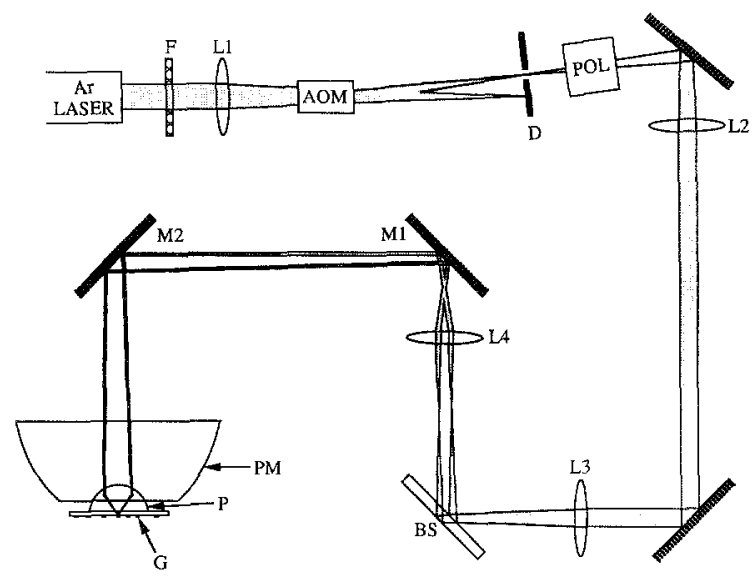

Fig. 6. Optical system for intersecting-beam TIRF. The illuminationsource is an argon ion laser (Coherent Innova 20; Coherent, Inc., Laser Products Division; Palo Alto, CA) operated at $488 \mathrm{~nm}$. The output power at the laser was $\sim 2 \mathrm{~W}$, followed by a $10 \%$ transmitting attenuator filter $(F)$. The laser intensity variation necessary to provide a bright photobleaching flash preceded and followed by a continuous dim probe illumination is computer-controlled by modulating the firstorder diffraction beam of an acoustooptic modulator (AOM). A crystal polarizer (POL) selected for p-polarization at the plane of TIR incidence because s-polarized light at an aluminum-solution interface produces an evanescent field of almost zero intensity [28]. Lens L3, placed before the beam splitter (BS), focuses the beam so that the two daughter beams converge to two points, which serve as objects for lens LA. Lens L4 places real images of these two points on, or near, the surface of the paraboloid mirror (PM; Optical Radiation Corp.; Azusa, CA). The BS is $1 \mathrm{~mm}$ thick and has a graded silvering on its front surface and a complete silvering on its back surface. This allows the relative power of the two daughter beams to be adjusted by sliding the BS laterally, normally so that there was equal power in each of the two daughter beams. Mirrors M1 and M2 direct the two daughter beams to near the bottom edge of the paraboloid mirror, which focuses and reflects the beams radially into the hemispherical prism $(\mathrm{P})$ and the polylysinated slide. The beams intersect and totally internally reflect at the aluminum-solution interface of the slide (at which the erythrocyte ghosts adhere), positioned near the focal point of the paraboloid mirror. In principle, this design allows the intersection angle and angle of incidence to be varied without moving the site of intersection. In practice in all these experiments, the intersection angle was $\sim 23^{\circ}$ and the angle of incidence was $\sim 72^{\circ}$. The intersecting beams produce an interference fringe pattern which was used in experiments designed to measure surface diffusion. If fringe pattern illumination is not desired, then one of the two beams can be blocked so that the sample is illuminated by a single beam. Other abbreviations are diaphragm (D), adhered erythrocyte ghosts (G), and lenses (L1 and L2).

a typical area from which fluorescence is transmitted by the image plane diaphragm.

\section{Data Acquisition}

TIR-FRAP data were acquired by a personal computer (80286 AT compatible) hosting a Metrabyte CTM-
05 counter/timer/IO PC board which counted photon pulses (preprocessed by an Ortec amplifier/discriminator system). A custom-written Fortran/assembly language program stored the counts in a chain of successive time intervals (or "bins"). During data acquisition, the Metrabyte board also issued the bleaching command pulse to the AOM. We used 200 prebleach and 800 postbleach bins (a "run") surrounding each bleaching flash, of bin durations of 1,10 , or $100 \mathrm{~ms}$. For single-beam experiments, the bleach durations were generally equal to or double the bin duration. For double-beam (striped fringe) experiments, the bleach duration was always set to half that for the corresponding single-beam experiment. To overcome shot noise, several consecutive photobleaching runs were performed on a single ghost and averaged together, to form a "set" of data. The time interval between successive runs ranged from 20 to $300 \%$ longer than the duration of a single run.

TIR-FRAP experiments were performed on the crescent areas of ghosts, which showed clear contrast between the crescent and the dimmer bite region. Although absolute intensities were quite variable from preparation to preparation (due in part to variations in metal film thickness), a typical probe intensity-excited photon count as seen through the square image plane diaphragm was $\sim 14,000 / \mathrm{s}$ in the crescent region, $\sim 8500$ / $s$ in the bite region, and $\sim 6000 / \mathrm{s}$ off-cell (which includes a contribution from the bulk solution).

To see the sample for positioning, we had to boost the illumination to a higher intensity than used for the TIR-FRAP probe intensity. Thus, during the search for a ghost in the crescent-bite configuration and the subsequent sample and illumination positioning, it is likely that most irreversibly adsorbed f-insulin that may have been present was photobleached before the actual photobleaching runs began; the results therefore accurately reflect the kinetics of reversible adsorption components on the time scale of a complete run only. After pos:tioning, we allowed the sample to recover for a few minutes in the dark, and then for a minute or two at the laser probe intensity, before TIR-FRAP runs were begun.

\section{Curve Fitting}

TIR-FRAP data (normalized to its prebleach fluorescence) was curve fit to gain information about the values of the desorption rate constants, $k_{\mathrm{d}}$, the relative weight of each, and a single surface diffusion coefficient $D_{\mathrm{s}}$. A Fortran subroutine essentially identical to the subroutine CURFIT by Bevington [25] was used to do the 


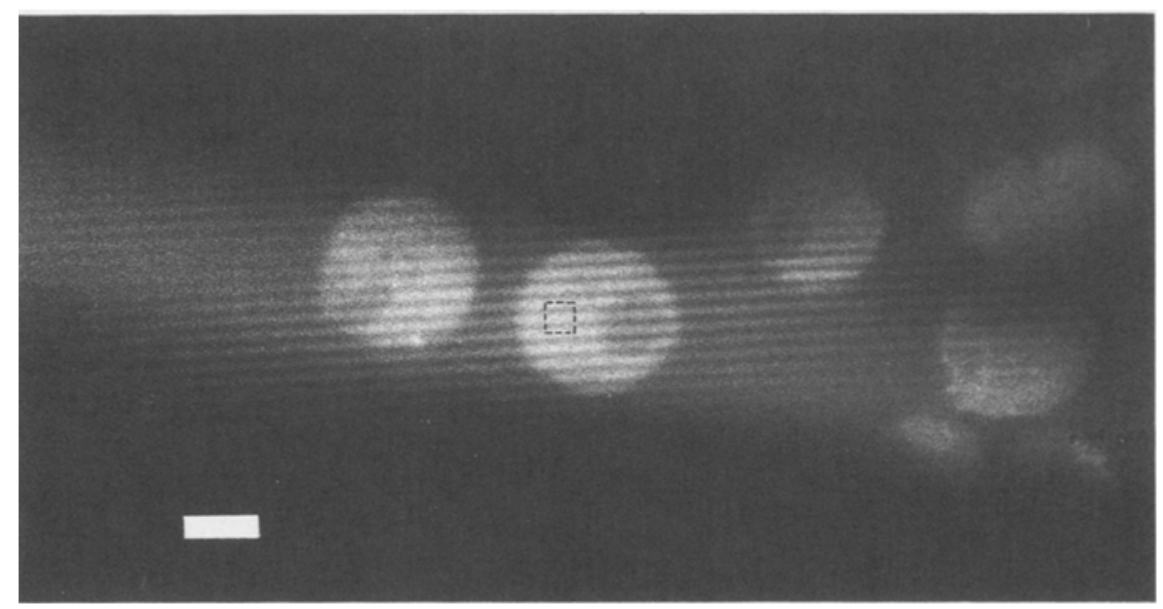

Fig. 7. Flattened erythrocyte ghosts under TIRF intersecting beam illumination as used here. The ghosts are in equilibrium with $50 \mu M$ fluorescein insulin, the same solution as used for all the quantitative TIR-FRAP experiments. The fringe pattern period is $0.90( \pm 0.02) \mu \mathrm{m}$. The photograph was taken through a $50 \times, 1.00$-NA water-immersion objective. The drawn-in outline shows a typical area from which light is accepted by the image plane diaphragm. Space bar, $5 \mu \mathrm{m}$.

curve fitting. We used two types of fitting functions. One type is just a simple sum of two exponentials:

$$
f_{N}(t)=1-\sum_{i=1}^{N} a_{i} \exp \left(-r_{i} t\right)
$$

with $N=2$, where the fitting parameters $a_{i}(\geq 0)$ are the amplitudes of the exponentials and the $r_{i}(\geq 0)$ are exponential rate constants. The fractional contribution $\alpha_{i}$ of each exponential is defined as $\alpha_{i} \equiv a_{i} / \Sigma_{i=1}^{N} a_{i}$. In cases where the kinetic rates are sufficiently slow, a double exponential, in principle, can correctly describe the recovery in a system with two kinetic processes working in parallel (i.e., two noninteracting classes of binding sites). But in practice, there may be additional bleaching during the probe illumination or incomplete recovery between successive bleaches. Therefore, Eq. (1) is used here mainly to characterize quantitatively the shape of a recovery curve in terms of the four numbers $\alpha_{1,2}$ and $r_{1,2}$. The connection between these fitting parameters and the physical constants $k_{\mathrm{d} i}$ and $D_{\mathrm{s}}$ is discussed in the Results section.

Another fitting function type (see Appendix), used only with single-beam illumination data, takes into account bleaching during probe illumination and incomplete recovery between successive bleaches and thereby directly yields estimates of actual desorption rate constants $k_{\mathrm{d} z}$ and their relative fractions of the adsorbed f-insulin.

Since "nonspecific" binding likely involves a heterogeneous set of surface targets with a (possibly con- tinuous) spread of kinetic rates, we defined a fit to be "acceptable" if it yielded a relative uncertainty of each fitting parameter of less than about $1 / 3$, and a normalized (reduced) chi-square, $\chi_{v}^{2}$, of less than 1.5. A wider set of rate constants can be deduced, however, by comparing results from different time scales, as described in the next section.

\section{RESULTS}

Analysis of the TIR-FRAP results had two goals: (1) to determine quantitatively adsorption/desorption kinetic rates (which are the reciprocals of the mean residence times on the surface) from the single-beam results and (2) to determine a range of possible surface diffusion coefficients $D_{\mathrm{s}}$ consistent from a comparison of the single- and double-beam TIR-FRAP results.

In TIR-FRAP, a recovery can be rate limited either by the adsorption/desorption kinetic rates (the "reaction limit") or by the bulk diffusion rate (the "bulk diffusion limit"). The latter rather uninteresting circumstance arises if the bulk diffusion is so slow or the kinetics so fast that a postbleach "cloud" of bleached fluorophore in the bulk fails to dissipate in a time shorter than the average time an insulin remains adsorbed to the surface. Which limit applies depends on the ratio of the surfacebound concentration to the bulk concentration at equilibrium [4]. An approximate quantitative estimate of the amount of nonspecific binding can be made [20] based 
on observed fluorescence intensities, near-metal quenching theory [13], and reasonable assumptions about the proximity of the ghost membranes from the Al-coated quenching surface. At the $50 \mu M$ bulk concentrations we used, we estimate 5000 nonspecific bindings of insulin $/ \mu \mathrm{m}^{2}$. From this result, we can make rough estimates of characteristic bulk normal diffusion-limited rates for the (f-insulin)-ghost system. None of these estimates are less than $10^{3} \mathrm{~s}^{-1}$, whereas the largest rate constant value derived from curve fitting our single-beam illumination "crescent" data is $270 \mathrm{~s}^{-1}$. Thus the (f-insulin)-ghost system is closer to the "reaction limit" [4] than the bulk diffusion limit, and the TIR-FRAP results thereby reflect actual adsorption/desorption kinetics.

\section{Desorption Rates: Single-Beam Experiments}

Table I shows the fitting parameters to the singlebeam illumination data for both the "exact" fitting function (column A) based on Eq. (A.5), which yields estimates of two desorption rate constants and the relative fractions of adsorbed $f$-insulin with each rate, and a simple double-exponential function (column B). It turns out that the fitted parameters for both functions are almost identical, suggesting that the corrections introduced by considering a finite time between successive bleaches and bleaching during probe illumination are very minor in our experiments.

Since the values obtained for desorption rate constants shown in Table I depend on the time scale (bin duration) of the experiment, there is probably a somewhat continuous distribution of desorption rate constants extending over a wider range than a single time scale can accommodate. To help characterize the nature of this distribution, we merged all of the data from all three time scales into one large data set. The merging was done by (a) fitting each time scale's averaged data $f$ (normalized to its prebleach value) to a multiexponential [Eq. (1)]; (b) forming the functions $g \equiv 1-f$ for the averaged data on each time scale; (c) multiplying and adding to the $g$ functions by-appropriate constants so the slopes and amplitudes of their fits match in regions of time overlap; and (d) fitting the resulting merged data to a sum of four exponentials as follows:

$$
g(t)=\sum_{i=1}^{4} a_{i} \exp \left(-r_{i} t\right)
$$

This merged $g(t)$ data and the curve resulting from this fit are shown in Fig. 8. Table II lists the exponential rate constants, $r_{i}$, and their respective fractional contributions, $\alpha_{i} \equiv a_{i} /\left(a_{1}+a_{2}+a_{3}+a_{4}\right)$.

Table I. Rate Parameters $r_{1,2}\left(\mathrm{~s}^{-1}\right)$ and Corresponding Fractions $\alpha_{1,2}$ for Insulin Adsorption onto the "Crescent" Portion of Flattened Erythrocyte Ghosts ${ }^{a}$

\begin{tabular}{|c|c|c|c|c|c|c|}
\hline \multirow{2}{*}{$\begin{array}{c}\text { Bin } \\
\text { duration } \\
\text { (ms) }\end{array}$} & \multirow[b]{2}{*}{ Parameter } & \multirow[b]{2}{*}{$\begin{array}{l}\text { No. runs/ } \\
\text { (No. ghosts) }\end{array}$} & \multicolumn{2}{|c|}{ Single beam } & \multicolumn{2}{|c|}{ Double beam } \\
\hline & & & $\begin{array}{c}\text { A } \\
\text { Eq. (A5) }\end{array}$ & $\frac{\text { B }}{\text { 2-exp. fit }}$ & $\begin{array}{l}\text { No. runs/ } \\
\text { (No. ghosts) }\end{array}$ & $\frac{C}{\text { 2-exp. fit }}$ \\
\hline \multirow[t]{4}{*}{100} & $r_{1}$ & $50 /(4)$ & $0.63 \pm 0.07$ & $0.64 \pm 0.06$ & $30 /(4)$ & $0.64 \pm 0.08$ \\
\hline & $\alpha_{1}$ & & $0.64 \pm 0.03$ & $0.64 \pm 0.04$ & & $0.67 \pm 0.04$ \\
\hline & $r_{2}$ & & $0.040 \pm 0.007$ & $0.042 \pm 0.006$ & & $0.033 \pm 0.004$ \\
\hline & $\alpha_{2}$ & & $0.36 \pm 0.03$ & $0.36 \pm 0.04$ & & $0.33 \pm 0.04$ \\
\hline \multirow[t]{4}{*}{10} & $r_{1}$ & $240 /(5)$ & $3.4 \pm 0.4$ & $3.5 \pm 0.4$ & $150 /(4)$ & $2.6 \pm 0.2$ \\
\hline & $\alpha_{1}$ & & $0.55 \pm 0.0$ & $0.56 \pm 0.03$ & & $0.57 \pm 0.04$ \\
\hline & $r_{2}$ & & $0.21 \pm 0.02$ & $0.23 \pm 0.02$ & & $0.20 \pm 0.02$ \\
\hline & $\alpha_{2}$ & & $0.45 \pm 0.02$ & $0.44 \pm 0.03$ & & $0.43 \pm 0.04$ \\
\hline \multirow[t]{4}{*}{1} & $r_{1}$ & $1200 /(1)$ & $38 \pm 5$ & $38 \pm 5$ & $1200 /(1)$ & $40 \pm 5$ \\
\hline & $\alpha_{1}$ & & $0.65 \pm 0.02$ & $0.65 \pm 0.02$ & & $0.58 \pm 0.02$ \\
\hline & $r_{2}$ & & $1.6 \pm 0.2$ & $1.6 \pm .2$ & & $1.5 \pm 0.1$ \\
\hline & $\alpha_{2}$ & & $0.35 \pm 0.02$ & $0.35 \pm 0.02$ & & $0.42 \pm 0.02$ \\
\hline
\end{tabular}

"Single-beam (uniform illumination) data were fit either by the "exact" theory of Appendix A (column A) or by a double exponential (column B). Double-beam (fringe pattern) data were fit by a double exponential (column C). The total number of runs and the number of separate ghosts are given for each optical configuration and time scale. For cases in which there is more than one data set, corresponding fitting parameters were averaged together and the standard deviations of the means were computed; these are the averages that are displayed. For cases in which there is only one data set, the listed uncertainties of the parameters are the values estimated by the curve-fitting program. For the great majority of these fits, $\chi_{v}^{2} \approx 1$. 


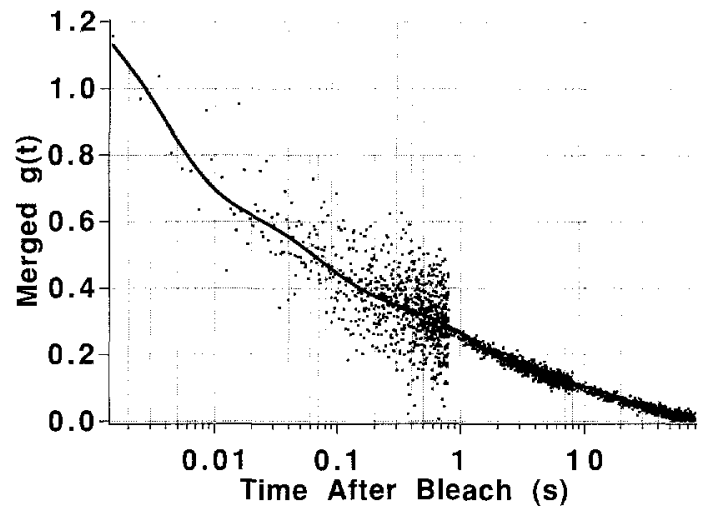

Fig. 8. Experimental TIR-FRAP data for f-insulin on erythrocyte membrane from three time scales, all merged into one continuous display as described in the text. Solid line is a four-exponential fit as described.

Table II. Exponential Rate Constants, $r_{i}$, and Their Respective Fractional Contributions, $\alpha_{i} \equiv a_{i} /\left(a_{1}+a_{2}+a_{3}+a_{4}\right)$ for f-Insulin Adsorbed to Erythrocyte Membrane, Corresponding to the Data in Fig. $8^{a}$

\begin{tabular}{ccc}
\hline Component $i$ & Fraction $\alpha_{i}$ & Rate $r_{i}\left(\mathrm{~s}^{-1}\right)$ \\
\hline 1 & $0.48 \pm 0.04$ & $270 \pm 60$ \\
2 & $0.23 \pm 0.02$ & $15 \pm 2$ \\
3 & $0.17 \pm 0.02$ & $0.77 \pm 0.07$ \\
4 & $0.12 \pm 0.01$ & $0.043 \pm 0.003$ \\
\hline
\end{tabular}

${ }^{a}$ Estimates of the uncertainty values for these parameters were based only on the photon shot noise in the data.

\section{Surface Diffusion and Fringe Pattern Results}

To determine a range of surface diffusion coefficients that is in agreement with our FRAP data, singlebeam illumination data were compared with fringe pattern illumination data (Table I, column C) as fit to a double exponential. Qualitatively, we would expect a somewhat faster double-beam recovery if surface diffusion were significant. A comparison of column $\mathrm{C}$ with column A or B shows that there is no evidence of a faster recovery.

Taking into account the uncertainty spread of the results, we can find an upper limit to the rate of surface diffusion. To do this, the four fit parameters $\alpha_{1,2}$ and $r_{1,2}$ are expressed as just one weighted average rate $W$ :

$$
W \equiv \alpha_{1} r_{1}+\alpha_{2} r_{2}
$$

Next we generate a series of artificial double-beam curves with a range of hypothetical single surface diffusion coefficients $D_{\mathrm{s}}$, using Eq. (A7), which takes into account possible bleaching during probe illumination. Generation of these curves requires that we input the known values of the interfringe spacing, the bleach-to-probe intensity ratio and the actual desorption rates and fractions from the single-beam data (Table I, column A or B) found on the same ghosts. These artificial double-beam curves are then characterized by curve fitting to the double-exponential form [Eq. (1) with $N=2$ ] followed by weighted averaging of the fit parameters [Eq. (3)]. The resulting "theoretical" weighted average rate $W_{\mathrm{T}}$, as a function of $D_{\mathrm{s}}$, is then compared with the "experimental" $W_{\mathrm{E}}$ (from Table I, column $\mathrm{C}$ ) to see at what values of $D_{\mathrm{s}}$, if any, might agreement be found. This comparison is displayed in Figs. 9a-c. The uncertainty bands for $W_{\mathrm{E}}$ and $W_{\mathrm{T}}$ were derived from the corresponding fitting parameter uncertainties using standard error propagation formulas. The details of these procedures can be found in Fulbright [20].

Figure 9a shows the results for the $100-\mathrm{ms}$ bin duration. The simulated curve is relatively flat, showing little sensitivity to $D_{\mathrm{s}}$. This is to be expected because the bleach depth parameters used to generate these curves were extremely large so that (in the model) even fluorophores in "dark" regions of the fringe pattern are mostly bleached, leaving few unbleached fluorophores on the surface to contribute to the fluorescence recovery via surface diffusion. Since the curves from the simulated data agree with the experimental values for all plotted $D_{\mathrm{s}}$ values, no conclusions can be drawn about possible values of $D_{\mathrm{s}}$.

Figure $9 \mathrm{~b}$ shows the results from the $10-\mathrm{ms}$ bin duration data. The simulated curves show considerably more sensitivity to $D_{\mathrm{s}}$ than the corresponding $100-\mathrm{ms}$ bin duration curves, due to the lower values of the bleach depth parameter. These curves show that, in principle, it is possible to resolve a range of surface diffusion coefficients using this technique. Only a surface diffusion coefficient of $<1 \times 10^{-10} \mathrm{~cm}^{2} / \mathrm{s}$ comes close to agreeing with the experimental fringe pattern data. In comparing single- and double-beam results for 10 -ms bin duration, we wished to exclude the possibility of bias due to some sort of photochemical damage imprinted by the first series of experiments. Therefore, we separately analyzed data from ghosts on which the single-beam experiments were performed before vs after the fringe pattern experiments or on which no fringe pattern illumination experiments were performed at all. No essential difference was noted: the only $D_{\mathrm{s}}$ compatible with the results is $<1 \times 10^{-10} \mathrm{~cm}^{2} / \mathrm{s}$ in all cases. 

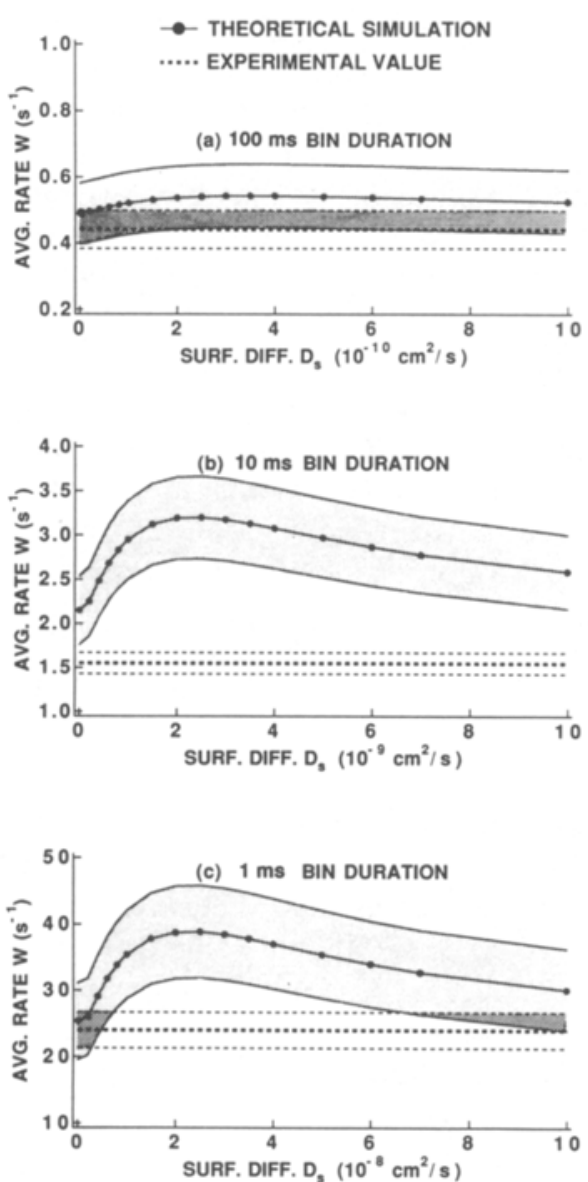

Fig. 9. Comparison of the weighted averages of rate constants derived from curve fitting (i) the fringe-pattern TIR-FRAP data (dashed lines) with (ii) simulated data generated from the actual desorption rates as measured in single-beam TIR-FRAP and a range of postulated surface diffusion coefficients (solid lines), as described in the text. The thinner lines represent the uncertainty bounds for each. Results from different photon-counting bin durations are shown: (a) $100 \mathrm{~ms}$; (b) $10 \mathrm{~ms}$; (c) $1 \mathrm{~ms}$.

Figure $9 \mathrm{c}$ shows the weighted average of rate constants results for the 1-ms bin duration. All of the 1-ms bin duration data were taken from the same ghost and the fringe pattern illumination data were taken before the single-beam illumination data. Figure $9 \mathrm{c}$ shows that $D_{\mathrm{s}}$ values in the approximate range $7 \times 10^{-9} \mathrm{~cm}^{2} / \mathrm{s}<D_{s}<$ $6.6 \times 10^{-8} \mathrm{~cm}^{2} / \mathrm{s}$ are not in agreement with the data. This excluded range of possible $D_{\mathrm{s}}$ values is consistent with the results from the $10-\mathrm{ms}$ bin duration data in that some $D_{\mathrm{s}}$ values $>10^{-10} \mathrm{~cm}^{2} / \mathrm{s}$ are excluded. Low signal-tonoise probably prevents more values in the plotted $D_{\mathrm{s}}$ range from being excluded.

\section{DISCUSSION}

The main purposes of this study were (a) to characterize the largely unexplored field of nonspecific binding of peptide hormones to cell membranes in terms of binding strength, reversibility, and surface diffusion; (b) to judge whether nonspecific binding might enhance :eaction rates with specific receptors; and (c) to introduce generally useful techniques of fluorescence, membrane preparation, and data analysis for studying molecular adsorption dynamics.

\section{Nonspecific Binding Rates and Equilibrium Constants}

We find that nonspecific binding of insulin to cell membranes (at the high bulk concentration of $50 \mu \mathrm{M}$ used here) is observable by TIRF microscopy, and we estimate about 5000 nonspecific bindings of insulin $/ \mu \mathrm{m}^{2}$. According to Gambhir et al. [10], a bulk insulin concentration of only $150 \mathrm{n} M$ is sufficient for occupying about half ( 7 of the $14 / \mu \mathrm{m}^{2}$ ) of the specific insulin receptor sites on human erythrocytes. (The prevailing concentration in the blood ranges from $10^{-11}$ to $10^{-8} \mathrm{M}$ [26].) At that $150 \mathrm{n} M$ bulk concentration, our extrapolated nonspecific binding results would predict a minimum nonspecific surface concentration of $\sim 10$ insulin molecules $/ \mu \mathrm{m}^{2}$. Therefore, total nonspecific binding, which includes fairly rapidly reversible fractions, is comparable to or greater than the specific binding at all bulk concentrations, including physiological concentrations. If nonspecifically bound insulin could also surface diffuse, then it is reasonable to conjecture that the nonspecific binding might play a role in specific binding rates to receptors. Whether it does or not depends upon the mean time of adsorption and the surface diffusion coefficient.

The mean time of adsorption (the reciprocal of the dissociation rate constant) of nonspecifically bound insulin covers a wide and apparently continuous range from about $4 \mathrm{~ms}$ to $25 \mathrm{~s}$, with the distribution skewed more heavily toward shorter times. The long value of $25 \mathrm{~s}$ is probably an underestimate, because even longer-time (more irreversible) binding tends to become photobleached during observation.

We find evidence that the average surface diffusion rate of $\mathrm{f}$-insulin is $<1 \times 10^{-10} \mathrm{~cm}^{2} / \mathrm{s}$. However, it is possible that some small fraction (presumably the most loosely bound and rapidly reversible fraction) may still surface diffuse and escape our detection. 


\section{Reaction Rate Enhancement}

If we assume that the nonspecific binding was far from saturating the surface, then the equilibrium of 50 $\mu M$ in the bulk and 5000 insulins $/ \mu \mathrm{m}^{2}$ on the surface gives an equilibrium constant in the reaction of bulk $\leftrightarrow$ surface of $150 \mathrm{~nm}$. Given the equilibrium constant of $150 \mathrm{~nm}$ for nonspecific binding and the dissociation rates $k_{\mathrm{d}}$ of 0.04 to $270 \mathrm{~s}^{-1}$ measured here, we can use the theory of Wang et al. [3] to determine what surface diffusion coefficient would be necessary to result in reaction rate enhancement with specific receptors. From Fig. 8 of that paper, it is clear that the ability of surface diffusion to enhance kinetic rates increases with $k_{\mathrm{d}}$. At the maximum $k_{d}$ we observe $\left(270 \mathrm{~s}^{-1}\right)$, the surface diffusion coefficient $D_{\mathrm{s}}$ would have to be at least $1.5 \times$ $10^{-8} \mathrm{~cm}^{2} / \mathrm{s}$ to be efficacious.

As can be seen from Figs. 9b and $\mathrm{c}$ here, any surface diffusion coefficients between about $1 \times 10^{-10}$ and $7 \times 10^{-8} \mathrm{~cm}^{2} / \mathrm{s}$ seem inconsistent with our data. At the slower end of this range, the theory of Wang et al. [3] clearly rules out any significant contribution to specific binding kinetics. Beyond the higher end of the range, our techniques could conceivably have missed surface diffusion of the order of $1 \times 10^{-7} \mathrm{~cm}^{2} / \mathrm{s}$. A $D_{\mathrm{s}}$ that large is possible in principle. The bulk diffusion coefficient for insulin free in aqueous solution is $D=15 \times 10^{-7}$ $\mathrm{cm}^{2} / \mathrm{s}$ [26]; one might expect that insulin adsorbed to the erythrocyte surface, which is irregular on a molecular scale and coated with entangled charged sugar chains, would suffer a significantly slowed surface diffusion. Therefore, our results suggest that "reduction of dimensionality" enhancement of kinetic rate-i.e., nonspecific adsorption followed by surface diffusion-is not operant for insulin on the erythrocyte surface. Nevertheless, surface diffusion of certain proteins, notably bovine serum albumin, on certain artificial surfaces has been detected by TIR-FRAP $[5,7]$. Therefore, it is still entirely possible that the interaction rate of certain bulk proteins with specific cell surface receptors on some cell types might benefit from surface diffusion.

\section{Techniques}

A number of novel experimental methods are employed here which may find potential uses in future studies of molecular dynamics at biological surfaces.

These experiments show that TIR-FRAP optics in a microscope configuration can detect weak nonspecific binding and desorption kinetic rates of fluorescent adsorbates on a biological membrane. Detection of surface diffusion is also possible in principle, but inclusion or exclusion of a given range of surface diffusion coefficients may require a higher signal/noise in the original data than measurement of desorption rates. This requirement arises from the fact that even the most optimal case-100\% surface diffusion of all adsorbates over the interfringe spacing distance before desorption-leads to a fluorescence recovery of only one-third of the bleaching depth [27], whereas adsorption/desorption kinetics produces a full recovery. Therefore the maximum possible "signal" in searching for surface diffusion is somewhat less than that for adsorption/desorption kinetics. This problem can be removed by use of a single small spot or stripe for photobleaching and probe illumination, but in that case it is more difficult to produce as small a characteristic diffusion distance or to gather as much fluorescence.

The specially derivatized aluminum coating used here can suppress background fluorescence due to adsorption directly to the substrate while preserving fluorescence from membrane surfaces only tens to hundreds of nanometers away. Since the aluminum coating could be derivatized by organosilanes (here for subsequent covalent attachment of polylysine), this surface preparation is a general method for selective quenching within distances of less than $10 \mathrm{~nm}$ from a chemically functionalized surface. The aluminum coating, at about $30-\mathrm{nm}$ thickness, is almost opaque to normally transmitted light. However, TIR illumination can take advantage of the high incident throughput at the surface plasmon angle $[13,28]$, which is a few degrees higher than the TIR critical angle. In a narrow range around the surface plasmon angle, the evanescent field intensity can be an order of magnitude higher than the incident intensity.

The problem of heating on an aluminum-coated surface during the bleaching pulse can be significant, since some of the incident light energy is turned into heat in the nonperfect metal, as represented by the imaginary (dissipative) part of the metal's dielectric constant. For the conditions of our experiments, we calculate from a very approximate theory [20] that a greatly overestimated upper bound for the temperature rise during the photobleaching flash is about $40^{\circ} \mathrm{C}$. The overestimation arises from the assumption of this approximation that the heat dissipates away only by direct conduction through the water and glass and thereby ignores the rapid conduction through the metal. A more exact solution is mathematically very difficult but may be warranted. As a matter of practice, one can approach the bleaching intensity that just causes instaneous local boiling, then scale back the incident bleaching intensity by at least a factor of 10 .

Perhaps the most startling technical feature of these 
experiments is the distinct and orderly manner in which erythrocytes flatten out and split open upon hemolysis on a surface with covalently attached polylysine. The clear and recognizable separation of external surface ("crescent") and cytoplasmic surface ("bite") regions facing the solution may be useful for other studies. This preparation may be particularly promising for studying the chemical kinetics of protein filaments at the cytoplasmic face.

The experimental production of interference fringes by intersecting TIRF beams is not new [29], nor is the use of non-TIR interference fringes for FRAP [30]. A theory for the combination of TIR with interference fringes has been presented [27]. The combination is used experimentally here, and in a configuration whereby the interfringe spacing and the incidence angle can be adjusted, while the area of illumination remains fixed relative to the microscope optical axis and random scattering is minimal. In practice, the optical setup is nontrivial and requires fine (and expensive) small translators. A less flexible and more highly scattering, but more convenient and mechanically stable method for producing intersecting TIR beams can be instituted by a prismless and mirrorless epiillumination method [31].

Finally, the data analysis technique used here yields the range of diffusion coefficients compatible with results, while taking into account complicating effects such as slight bleaching during the probe phase of the experiments and rapidly repetitive bleaching before complete recovery. One can show $[6,20]$ the rather counterintuitive result that bleaching during the probe phase always increases the apparent recovery rate.

In summary, we have used a novel configuration and sample configuration of TIR-FRAP to examine adsorption/desorption kinetics and surface diffusion of a fluorophore-labeled hormone at a biological cell surface.

\section{APPENDIX}

Here we present TIR-FRAP theoretical recovery functions for single-beam (uniform) and double-beam (fringe pattern) illumination. (For details of the derivations, see Fulbright [20].) The single-beam form corrects for both bleaching during probe illumination and for incomplete recoveries in a sequence of successive signal averaged bleach recoveries. The double-beam form only corrects for bleaching during probe illumination. In both cases, we assume that the system, represented by

$$
A \text { (bulk) } \underset{k_{\mathrm{d}}}{\stackrel{k_{\mathrm{a}}}{\rightleftarrows}} C \text { (adsorbed) }
$$

is in the "reaction limit" (in which the adsorption kinetics rather than bulk diffusion are rate-limiting; see Thompson et al. [4]) and that bleaching is an irreversible first-order process.

The single- and double-beam cases both are derived from solutions of initial value problems, which have the basic form:

$$
\begin{aligned}
\frac{\partial C(\vec{x}, t)}{\partial t}= & D_{\mathrm{s}} \nabla^{2} C(\vec{x}, t)-k_{\mathrm{d}} C(\vec{x}, t) \\
& +k_{\mathrm{\alpha}} A-\alpha P I(\vec{x}) C(\vec{x}, t) \\
C(\vec{x}, 0)= & C(\vec{x},-) \exp (-\alpha B I(\vec{x}) T)
\end{aligned}
$$

$C(\vec{x}, t)$ is the surface concentration of unbleached fluorophores at position $\vec{x}$ and time $t$ [where $t \geq 0$ after the bleach and $t=(-)$ before the bleach]. $D_{\mathrm{s}}$ is the surface diffusion coefficient of the adsorbed molecules. $A$ is the bulk concentration of fluorophores which, in the reaction limit, is assumed to be all unbleached and independent of distance from the surface. $P I(\vec{x})$ and $B I(\vec{x})$ are the probe and bleach illumination intensities, respectively, at position $\vec{x}$, with $I(\vec{x})$ being a unitless normalized profile function. $\alpha$ is proportional to the quantum efficiency of bleaching. $T$ is the bleach pulse duration.

To simplify notation, define the bleach depth parameter $K \equiv \alpha B T$ and the ratio of bleach illumination intensity to probe illumination intensity $\beta \equiv B / P$. Le $f_{i}$ be the fraction of molecules on the surface which have desorption rate constant $k_{\mathrm{d}}$, with $i$ ranging from 1 to $\mathrm{m}$. Also, define the functions $\Psi_{i}(t)$ and $\Omega_{i}(t)$ :

$$
\begin{aligned}
& \Psi_{i}(t) \equiv \frac{f_{i}}{\left(1+\frac{K}{T \beta k_{\mathrm{d} i}}\right)}\left\{1-\exp \left[-\left(k_{\mathrm{d}_{i}}+\frac{K}{T \beta}\right) t\right]\right\} \\
& \Omega_{i}(t) \equiv \exp (-K) \exp \left[-\left(k_{\mathrm{d} i}+\frac{K}{T \beta}\right) t\right]
\end{aligned}
$$

It can be shown [20] that after many successive bleaches regularly spaced in time by an interval $\tau$, the single-beam postbleach recovery of fluorescence $f(t)$ collected from the surface, normalized to its prebleach value, approaches the form

$$
f(t)=\frac{\sum_{i=1}^{m}\left[\Psi_{i}(t)+\Omega_{i}(t)\left(\frac{\Psi_{i}(\tau)}{1-\Omega_{i}(\tau)}\right)\right]}{\sum_{i=1}^{m}\left(\frac{\Psi_{i}(\tau)}{1-\Omega_{i}(\tau)}\right)}
$$

For double-beam (fringe pattern) illumination, the unitless profile function is 


$$
I(\bar{x})=1+\cos \left(q_{0} x\right)
$$

where the fringe pattern spatial period is $2 \pi / q_{0}$ and $x$ is a Cartesian position coordinate. [Note that, in general, fringes due to intersecting TIR evanescent fields will exhibit a lower contrast [27] but under our conditions, the contrast is close to the theoretical maximum as given in Eq. (A6).]

If the area of the surface from which fluorescence is collected (the observation area) is rectangular and oriented so that two of its sides are parallel to the fringe pattern, and if its length along the direction perpendicular to the fringes (the $x$ direction) is an integral number of fringe pattern periods, then the normalized collected postbleach fluorescence from the surface is given approximately by [20]

$f(t)=\frac{\sum_{i=1}^{m}\left[\Psi_{i}(t)+\gamma_{i} \Omega_{i}(t)\left[I_{0}(-K)+I_{1}(-K) \exp \left(-D_{\mathrm{s}} q_{0}^{2} t\right)\right]\right]}{\sum_{i=1}^{m} \gamma_{i}}$

where $I_{0}$ and $I_{1}$ are modified Bessel functions of the first kind of order 0 and 1 , respectively. We have found a simple expression for $\gamma_{i}$ only if the time interval between bleaching flashes, $\tau$, is much longer than $1 /\left(k_{\mathrm{d}_{i}}+\alpha P\right)$. Then $\gamma_{i}$ is given by

$$
\gamma_{i}=\frac{k_{\mathrm{d} i} f_{i}}{\left(k_{\mathrm{d} i}+(K / T \beta)\right)}
$$

\section{ACKNOWLEDGMENTS}

We thank Dr. Robert Petrisko for suggesting the silanization procedure for aluminum thin films, Drs. John Langmore and Michael Bretz for the use of their vacuum deposition chambers, Drs. Jon Aster and George Brewer for their gift of antibodies, Dr. Edward Hellen for his advice on metalized surface fluorescence quenching, and Dr. Andrea Stout for her help with data merging software. This work was supported by National Science Foundation Grant DMB 8805296 and National Institutes of Health Grant NS14565.

\section{REFERENCES}

1. G. Adam and M. Delbruck (1968) in A. Rich and N. Davidson (Eds.), Structural Chemistry and Molecular Biology, W. H. Freeman, San Francisco, pp. 198-215.

2. H. C. Berg, and E. M. Purcell (1977) Biophys. J. 20, 193-219.

3. D. Wang, S.-Y. Gou, and D. Axelrod (1992) Biophys. Chem. 43, 117-137.

4. N. L. Thompson, T. P. Burghardt, and D. Axelrod (1981) Biophys. J. 33, 435-454.

5. T. P. Burghardt and D. Axelrod (1981) Biophys. J. 33, 455-468.

6. E. H. Hellen and D. Axelrod (1991) J. Fluoresc. 1, 113-128.

7. R. D. Tilton, A. P. Gast, and C. R. Robertson (1990) Biophys. J. 58, 1321-1326.

8. M. L. Pisarchick, D. Gesty, and N. L. Thompson (1992) Biophys. $J ., 63: 215-223$.

9. K. H. Pearce, R. G. Hisket, and N. L. Thompson (1992) Biochemistry 31, 5983-5995.

10. K. K. Gambhir, J. A. Archer, and C. J. Bradley (1978) Diabetes 27, 701-708.

11. S. T. Sui, T. Urumow, and E. Sackmann (1988) Biochemistry 27, 7463-7469.

12. B. S. Jacobson, J. Cronin, and D. Branton (1978) Biochim. Biophys. Acta 506, 81-96.

13. E. H. Hellen and D. Axelrod (1987) J. Opt. Soc. Am. B 4, $337-$ 350.

14. R. R. Chance, A. Prock, and R. Silbey (1975) J. Chem. Phys. 62, 2245-2253.

15. R. R. Chance, A. Prock, and R. Silbey (1978) Adv. Chem. Phys. 37, 1-65.

16. M. R. Philpott (1975) J. Chem. Phys. 62, 1812-1817.

17. H. Morawitz and M. R. Philpott (1974) Phys. Rev. B 10, 4863 4868.

18. M. P. Sheetz and D. E. Koppel (1979) Proc. Natl. Acad. Sci. USA 76, 3314-3317.

19. C. S. Foote (1968) Science 162, 963-970.

20. R. M. Fulbright (1991) Ph.D. thesis, University of Michigan, Ann Arbor.

21. F. Tietze, G. E. Mortimore, and N. R. Lomax (1962) Biochim. Biophys. Acta 59, 336-346.

22. W. W. Bromer, S. K. Sheehan, A. W. Berns, and E. R. Arquilla (1967) Biochemistry 6, 2378-2388.

23. E. R. Arquilla, W. W. Bromer, and D. Mercola (1969) Diabetes 18, 193-205.

24. D. A. Mercola, J. W. S. Morris, and E. R. Arquilla (1972) Biochemistry 11, 3860-3874.

25. P. R. Bevington (1969) Data Reduction and Error Analysis for the Physical Sciences, McGraw-Hill, New York.

26. T. G. Blundell, D. Dodson, D. Hodgkin, and D. Mercola (1972) Adv. Prot. Chem. 26, 279-402.

27. J. R. Abney, B. A. Scalettar, and N. L. Thompson (1992) Biophys. J. 61, 542-552.

28. D. Axelrod, E. H. Hellen, and R. M. Fulbright (1992) in J. Lakowicz (Ed.), Topics in Fluorescence Spectroscopy, Vol. 3: Biochemical Applications, Plenum Press, New York, pp. 289343.

29. R. M. Weis, K. Balakrishnan, B. A. Smith, and H. M. McConnell (1982) J. Biol. Chem. 257, 6440-6445.

30. J. Davoust, P. F. Devaux, and L. Leger (1982) EMBO J. 1, 12331238.

31. A. L. Stout and D. Axelrod (1989) Appl. Opt. 28, 5237-5242. 